\title{
The trade-offs of cell over-provisioning in IEEE 802.15.4 TSCH networks
}

\author{
Fafoutis, Xenofon; Papadopoulos, Georgios Z.
}

\section{Published in:}

Proceedings of 17th International Conference on Ad Hoc Networks and Wireless

Link to article, DOI:

10.1007/978-3-030-00247-3_13

Publication date:

2018

Document Version

Peer reviewed version

Link back to DTU Orbit

\section{Citation (APA):}

Fafoutis, X., \& Papadopoulos, G. Z. (2018). The trade-offs of cell over-provisioning in IEEE 802.15.4 TSCH networks. In N. Montavont, \& G. Z. Papadopoulos (Eds.), Proceedings of 17th International Conference on Ad Hoc Networks and Wireless (pp. 132-137). Springer. Lecture Notes in Computer Science (including subseries Lecture Notes in Artificial Intelligence and Lecture Notes in Bioinformatics) Vol. 11104 LNCS https://doi.org/10.1007/978-3-030-00247-3_13

\section{General rights}

Copyright and moral rights for the publications made accessible in the public portal are retained by the authors and/or other copyright owners and it is a condition of accessing publications that users recognise and abide by the legal requirements associated with these rights.

- Users may download and print one copy of any publication from the public portal for the purpose of private study or research.

- You may not further distribute the material or use it for any profit-making activity or commercial gain

- You may freely distribute the URL identifying the publication in the public portal 
See discussions, stats, and author profiles for this publication at: https://www.researchgate.net/publication/327311647

The Trade-Offs of Cell Over-Provisioning in IEEE 802.15.4 TSCH Networks: 17th International Conference on Ad Hoc Networks and Wireless, ADHOC-NOW 2018, Saint-Malo, France, Septembe...

Chapter in Lecture Notes in Computer Science · August 2018

DOI: 10.1007/978-3-030-00247-3_13

CITATIONS

READS

0

34

2 authors:

Xenofon Fafoutis

Technical University of Denmark

89 PUBLICATIONS 975 CITATIONS

SEE PROFILE

IMT Atlantique

66 PUBLICATIONS 495 CITATIONS

SEE PROFILE

Some of the authors of this publication are also working on these related projects:

Wireless Sensor Networks (WSNs) View project

IRC SPHERE View project 


\title{
The Trade-offs of Cell Over-Provisioning in IEEE 802.15.4 TSCH Networks
}

\author{
Xenofon Fafoutis ${ }^{1,2, \star}$ and Georgios Z. Papadopoulos ${ }^{3}$ \\ 1 Department of Electrical and Electronic Engineering, \\ University of Bristol, Woodland Road, BS8 1UB, UK \\ xenofon.fafoutis@bristol.ac.uk \\ 2 DTU Compute, Technical University of Denmark, \\ Richard Petersens Plads, 2800 Kongens Lyngby, Denmark \\ xefa@dtu.dk \\ 3 IMT Atlantique, IRISA, UBL, France \\ georgios.papadopoulos@imt-atlantique.fr
}

\begin{abstract}
Wireless industrial applications require high level of Quality of Service (QoS) such as low-delay and jitter performances, low-power operations as well as end-to-end reliability close to $100 \%$. However, considering the large number of wireless networks operating in $2.4 \mathrm{GHz}$, the radio technologies are more prone to the external interference, which eventually may negatively affect the reliability, the delay and the goodput performance due to collisions and retransmissions. To tackle the previously detailed issues, Time-Slotted Channel Hopping (TSCH) Medium Access Control (MAC) protocol emerged with IEEE 802.15.4-2015 as an alternative to the industrial standards such as WirelessHART and ISA100.11a. TSCH is based on frequency hopping to avoid the interference, while the medium access is based on a scheduler (i.e., slotframe) that repeats periodically to avoid the collisions. Yet, the majority of the proposed TSCH schedulers are based on traditional collision detection and retransmission in the following slotframe, which essentially increases the end-to-end delay performance. In this poster, we consider allocating consecutive timeslots for a single data transmission, to allow thus, to retransmit the data packet within the slotframe in case of losses. We study the potential trade-offs, reliability and delay versus energy consumption, when considering the over-allocation approach.
\end{abstract}

Keywords: Internet of Things · IEEE 802.15.4 - Time-Slotted Channel Hopping · $6 \mathrm{TiSCH} \cdot$ Industrial Networks · Scheduling · Over-allocation.

\section{Introduction}

Industrial automation networks require end-to-end reliability above $99.9 \%$ and guaranteed worst case delay [7]. In 2016, IEEE 802.15.4-2015 standard [1] was

\footnotetext{
${ }^{\star}$ Work done prior to joining DTU Compute.
} 
published to provide Quality of Service guarantees. Among the Medium Access Control (MAC) protocols defined in the standard, Time-Slotted Channel Hopping (TSCH) is the scheme that is especially designed for the Internet of Things (IoT). A TSCH-based schedule is a matrix that consists of timeslots and channel offsets, where each square (i.e., cell) is an transmission opportunity.

$\mathrm{TSCH}$ has attracted significant attention from the industrial and research community as it may provide low-power and low-delay operation, as well as reliable and predictable wireless communication. Indeed, TSCH has been implemented in several IoT-based Operating Systems (OSs) ${ }^{4,5}$ and it has been already employed in number of real-world use cases, e.g. [3]. Furthermore, 6TiSCH Working Group (WG) was established in 2013 at the Internet Engineering Task Force (IETF) to enable IPv6 over the TSCH mode of IEEE 802.15.4 .

A number of TSCH-based schedulers have been proposed to improve the network performance, i.e., minimize the latency or to reduce the energy consumption [8]. Indeed, to further improve reliability, some works consider cell over-provisioning [5], when it is observed that the reliability between a pair of nodes drops a certain threshold, then the schedule may over-allocate extra cells to transmit the frames [10]. In that case, over-provisioning allows to improve the network reliability, and bound the end-to-end delay by allocating more cells to each radio link, but has a negative impact on both the energy efficiency and the network capacity.

In this work, we study the impact of introducing cell over-provisioning, i.e., multiple consecutive opportunities to transmit a single data packet. To that aim, we study a TSCH-based wireless network over various link qualities, to identify the minimum required number of cells per use-case. Our simulation results show that there is trade-off between the number of allocated slots and performance metrics, such as network reliability, delay and energy consumption. Since, the number of allocated cells in a static TSCH schedule affects the networks performance: under low link qualities, multiple retransmissions are necessary to transmit per data packet, which eventually impacts the energy consumption.

\section{Background and Motivation}

\subsection{IEEE 802.15.4-TSCH}

TSCH is based on Time Division Multiple Access (TDMA) and Frequency Hopping Spread Spectrum (FHSS) schemes. Indeed, it forms a schedule that is a matrix of timeslots ( $\chi$ axis) and channel offsets ( $\psi$ axis) to achieve high level of reliability by mitigating, through channel hopping, the impact of the external interference. The standard comes with 101 timeslots, which is one slotframe, and 16 channel offsets, thus, the maximum throughput is 1616 transmissions. In Fig. 1, a TSCH instant is illustrated. Similarly to TDMA, in TSCH the time

\footnotetext{
${ }^{4}$ https://github.com/contiki-ng/contiki-ng/wiki

${ }^{5}$ https://openwsn.atlassian.net/wiki/spaces/OW/overview

${ }^{6}$ https://datatracker.ietf.org/wg/6tisch/about/
} 


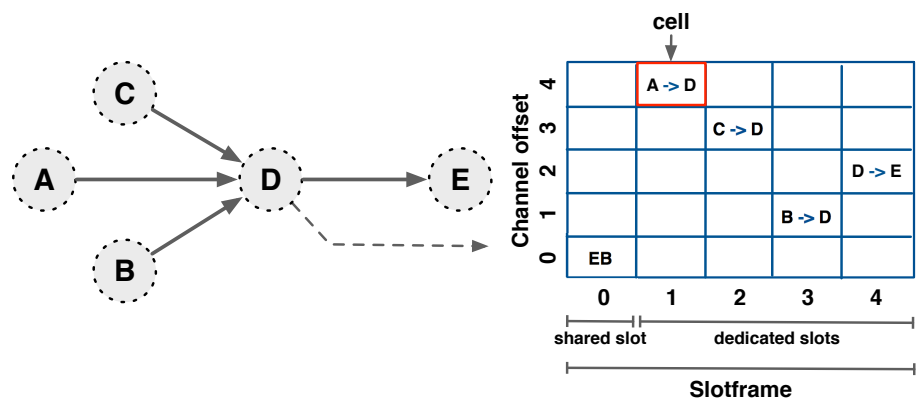

Fig. 1: An example of TSCH scheduling for node D. A $\rightarrow$ D stands for ' $A$ transmits to $D$ '. EB cells are used for broadcast and advertisement frames.

is slotted into timeslots of equal length, typically $10 \mathrm{~ms}$. At each timeslot, a node may transmit a frame and receive an acknowledgement. To achieve such communication, nodes' clocks should be constantly synchronized. A group of timeslots consists a slotframe that repeats perpetually. Each timeslot curries an Absolute Sequence Number (ASN), an integer value that counts the number of timeslots since the beginning of the TSCH network. Finally, the channel offset is translated into a physical radio channel through a Frequency Hopping Spread Spectrum (FHSS) algorithm.

\section{$2.2 \quad$ Related Work}

In 6TiSCH WG, the 6TiSCH Experimental Scheduling Function (SFX) [2] considers an over-provisioning algorithm to estimate the number of cells to be allocated. However, the term "over-provisioning" in SFX means to allocate additional cells for extra traffic, and not as conditional retransmission in consecutive timeslots of the same data packet.

In [5], the authors propose Adaptive Static Scheduling to allow, in a fully distributed manner, each pair of nodes to control their active timeslots in order to improve the energy efficiency of TSCH networks. A static scheduler is build at compilation time with excessive timeslot overallocation; yet, the nodes can dynamically activate or deactivate their a priori allocated timeslots, according to the traffic requirements. However, the authors did not consider the cost of blind over-provisioning in terms of delay performance.

In [10], the authors investigate the radio channel blacklisting issues. They consider an over-provisioning scheme to allocate additional timeslots per link to bound the end-to-end delay and to improve the network reliability in the case of losses. However, they did not study the impact of over-provisioning in terms of energy consumption.

In this work, we investigate the cost of cell over-provisioning depending on the link quality. Indeed, we highlight the potential trade-offs between network reliability and latency versus energy consumption. 


\section{Performance Evaluation}

To evaluate the impact of the cells over-allocation in a TSCH networks, we employ an open-source numerical TSCH Simulator [4]. This tools simulates starbased TSCH networks with arbitrary TSCH schedules. For each radio link, the simulator allows to configure parameters, such as the maximum number of retransmissions per frame, the queue size, and the link-layer packet reception probability. The simulator supports both contention-free (dedicated) timeslots and contention-based (shared) slots. In this study, we employ the contention-free timeslots only. The simulation parameters are provided in Table 1. For evaluating the energy consumption, we use the measurements on the GINA mote [6], presented in [9].

Table 1: Simulation Parameters

\begin{tabular}{|l|l|}
\hline Parameter & Value \\
\hline \hline Frame Size & 100 slots \\
\hline Duration of Simulation & 100 frames \\
\hline Max. Retransmissions & 8 \\
\hline Max. Queue Size & 8 \\
\hline No. of Sender Nodes & 4 \\
\hline Traffic Rate & $2-4$ ppf \\
\hline Packet Reception Rate (PRR) & $0.5-0.9$ \\
\hline Energy Efficiency Exponent & 1.2 \\
\hline
\end{tabular}

The TSCH Simulator provides results on Packet Delivery Rates (PDR), energy consumption, and energy efficiency defined as the energy required per successfully delivered packet. The latency is evaluated analytically as follows. We denote as $k$ the level of overallocation. Each transmission is modeled as a independent Bernoulli trial with the same probability of success, $p$ (i.e. PRR), for each trial. For simplicity, we assume infinite retransmissions. The probability that a packet will be successfully delivered after $i$ failed attempts is given by:

$$
q_{i}=(1-p)^{i} p .
$$

Focusing on the worst case scenario, we consider the $N$-th node and assume that the packet is generated at beginning of the frame. If the transmission occurs on the $i$-th attempt, the delay is given by the following equation:

$$
d_{i}=k N\left\lfloor\frac{i}{k}\right\rfloor+i \bmod k+k(N-1) .
$$

where $k(N-1)$ corresponds to the delay due to timeslots allocated to the other $N-1$ nodes in the first slotframe, $k N\left\lfloor\frac{i}{k}\right\rfloor$ corresponds to the delay of whole slotframes (when $i \geq k$ ), and $i \bmod k$ corresponds to the delay due to failed 


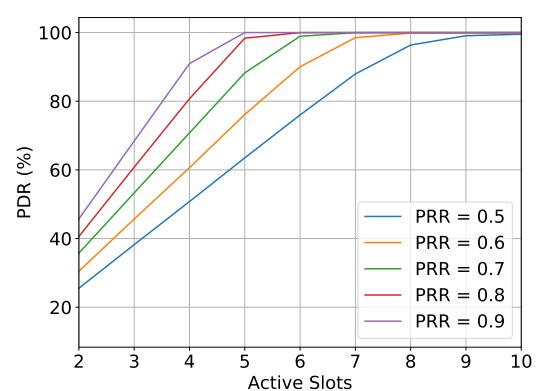

(a) Packet Delivery Rate (PDR)

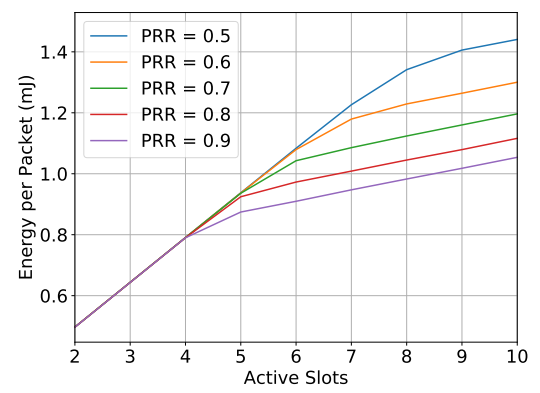

(c) Energy Consumption

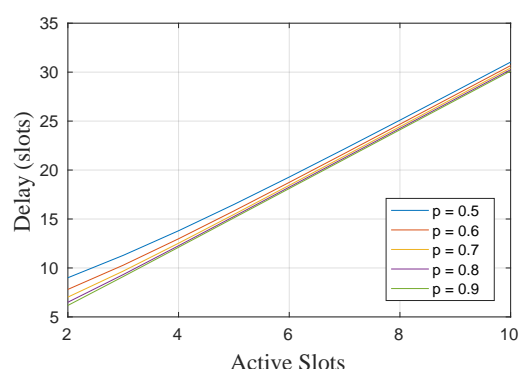

(b) Link Delay

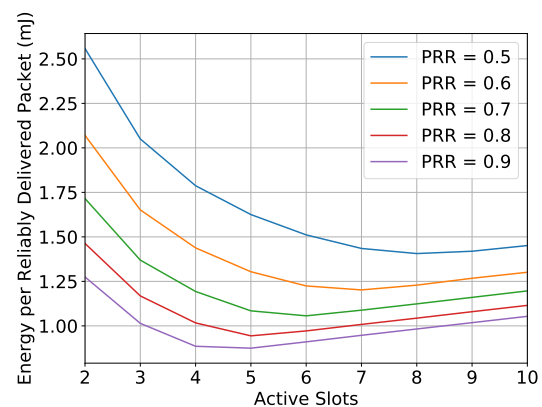

(d) Energy Efficiency

Fig. 2: TSCH Performance for various link qualities.

attempts in the last slotframe. The average delay is calculated as the probabilitybased weighted sum of $d_{i}$, and it is given by:

$$
D=\sum_{i=0}^{\infty} q_{i} d_{i}
$$

Fig. 2 plots the the reliability (PDR), delay and average energy consumed per packet of the TSCH network, respectively, for various numbers of active slots per sender per frame.

As it can be observed from the graphs, a trend shift strongly depends on PRR. Indeed, the lower is the PRR value the more active timeslots are necessary to achieve $100 \%$ of network reliability (i.e., Fig. 2(a)), which in turn affects the delay performance due to retransmissions (i.e., Fig. 2(b)) and increases the energy consumption, see Fig. 2(c). Furthermore, in Fig 2(d), we plot the energy per reliably delivered packets, which is a metric that combines reliability and energy consumption. It is observed that increasing the level of cell overprovisioning, has a positive effect on PDR, yet has a negative effect on both delay, due to the other nodes' overprovisioned cells, and energy consumption, 
due to the increased amount of idle listening. It is also observed that there is an optimization point for the energy per reliably delivered bit, demonstrating a trade-off between reliability and energy consumption.

\section{Conclusions}

TSCH employs frequency hopping to combat the potential external interference, however it is still insufficient. Therefore, to further improve the network reliability, TSCH schedules rely on overallocation to allow multiple retransmissions within the slotframe. The level of cell overallocation in TSCH-based schedules, however, affects the network performance. Indeed, we observe a strong tradeoff between reliability and delay versus energy consumption. In this poster, we investigate the costs of allocating additional timeslots per data packet.

\section{References}

1. IEEE Standard for Low-Rate Wireless Personal Area Networks (LR-WPANs). IEEE Std 802.15.4-2015 (Revision of IEEE Std 802.15.4-2011) (April 2016)

2. Dujovne, D., Grieco, L., Palattella, M., Accettura, N.: 6TiSCH Experimental Scheduling Function (SFX). draft, IETF (March 2018)

3. Elsts, A., Oikonomou, G., Fafoutis, X., Piechocki, R.: Internet of Things for smart homes: Lessons learned from the SPHERE case study. In: Global Internet of Things Summit (GIoTS). pp. 1-6 (June 2017). https://doi.org/10.1109/GIOTS.2017.8016226

4. Elsts, A., Fafoutis, X., Pope, J., Oikonomou, G., Piechocki, R., Craddock, I.: Scheduling High-Rate Unpredictable Traffic in IEEE 802.15.4 TSCH Networks. In: 13th Int. Conf. on Distributed Comput. in Sensor Syst. (DCOSS) (2017)

5. Fafoutis, X., Elsts, A., Oikonomou, G., Piechocki, R., Craddock, I.: Adaptive Static Scheduling in IEEE 802.15.4 TSCH Networks. In: Proceedings of the 4th IEEE World Forum on Internet of Things (WF-IoT) (2018)

6. Mehta, A.M., Pister, K.S.J.: WARPWING: A complete open source control platform for miniature robots. In: 2010 IEEE/RSJ International Conference on Intelligent Robots and Systems. pp. 5169-5174 (Oct 2010). https://doi.org/10.1109/IROS.2010.5649382

7. Papadopoulos, G.Z., Matsui, T., Thubert, P., Texier, G., Watteyne, T., Montavont, N.: Leapfrog Collaboration: Toward Determinism and Predictability in IndustrialIoT applications. In: Proceedings of the IEEE International Conference on Communications (ICC) (2017)

8. Rodrigo Teles Hermeto, A.G., Theoleyre, F.: Scheduling for IEEE802. 15.4-TSCH and Slow Channel Hopping MAC in Low Power Industrial Wireless Networks: A Survey. Elsevier Computer Communications 114, 84-105 (2017)

9. Vilajosana, X., Wang, Q., Chraim, F., Watteyne, T., Chang, T., Pister, K.S.J.: A Realistic Energy Consumption Model for TSCH Networks. IEEE Sensors Journal 14(2), 482-489 (Feb 2014). https://doi.org/10.1109/JSEN.2013.2285411

10. Zorbas, D., Kotsiou, V., Theoleyre, F., Papadopoulos, G.Z., Douligeris, C.: LOST: Localized Blacklisting Aware Scheduling Algorithm for IEEE 802.15.4-TSCH Networks. In: Proceedings of the 10th Wireless Days Conference (WD) (2018) 